\title{
Creation of strange-quark-matter droplets as a unique signature for quark-gluon-plasma formation in relativistic heavy-ion collisions
}

\author{
Carsten Greiner, Dirk-Hermann Rischke, and Horst Stöcker \\ Institut für Theoretische Physik der J. W. Goethe Universität Frankfurt, Postfach 111932, \\ D-6000 Frankfurt am Main 11, Germany \\ Peter Koch \\ Department of Physics, State University of New York at Stony Brook, Stony Brook, New York 11794-3800
}

(Received 22 January 1988)

\begin{abstract}
We demonstrate that strangeness separates in the Gibbs-phase coexistence between a baryon-rich quark-gluon plasma and hadron matter, even at $T=0$. For finite temperatures this is due to the associated production of kaons (containing $\bar{s}$ quarks) in the hadron phase while $s$ quarks remain in the deconfined phase. The $s-\bar{s}$ separation results in a strong enhancement of the $s$-quark abundance in the quark phase. This mechanism is further supported by cooling and net strangeness enrichment due to the prefreezeout evaporation of pions and $K^{+}, K^{0}$, which carry away entropy and antistrangeness from the system. Metastable droplets (i.e., stable as far as weak interactions are not regarded) of strange-quark matter ("strangelets") can thus be formed during the phase transition. Such cool, compact, long-lived clusters could be experimentally observed by their unusually small $Z / A$ ratio $(\leq 0.1-0.3)$. Even if the strange-quark-matter phase is not stable under strong interactions, it should be observable by the delayed correlated emission of several hyperons. This would serve as a unique signature for the transient formation of a quark-gluon plasma.
\end{abstract}

\section{INTRODUCTION}

The proposal that strange-quark-matter droplets ("strangelets") at zero temperature and in $\beta$ equilibrium might be absolutely stable ${ }^{1-4}$ has stimulated substantial activity. Such a scenario would have fundamental importance, for example, in cosmological models to explain a large fraction of the nonobservable mass of the Universe. $^{2}$ Recently we have proposed that such strange-quark-matter droplets might as well play an important role in the ultrarelativistic heavy-ion collisions, where one would expect a total strangeness of zero, high temperature and entropy, and no $\beta$ equilibrium. ${ }^{5}$

In the present paper it is shown how strangeness separates in the Gibbs-phase coexistence of a baryon-rich quark-gluon plasma and hadron matter, even at $T=0$ : The separation at $T=0$ does occur because the system gains energy, if the $s$ quarks remain in the quark phase. At finite $T$ the mechanism can be viewed as being due to the associated production of kaons (containing $\bar{s}$ quarks) in the hadron phase while the $s$ quarks reside in strangequark-matter droplets, which therefore have a net strangeness larger than zero. We show below that prompt kaon emission charges the system with finite net strangeness, leading to an even stronger enhancement of the $s$-quark abundance in the quark phase. Even more important, the quark phase actually condenses into energetically metastable droplets of strange-quark matter (i.e., they can only decay via weak interactions ${ }^{6}$ ) during the course of the expansion. ${ }^{5}$ The temperature and entropy of these strangelets drop dramatically from their initial values. They will then be emitted as long-lived $\left(\tau>10^{-4}\right.$ sec) (Ref. 6), nearly neutral clusters. Such strangelets, if they are actually formed, may serve as the only qualitatively new structure with an appropriate lifetime to prove the transient existence of a quark-gluon plasma.

This paper is organized in the following way. In Sec. II we study extensively the properties of strange-quark matter at zero temperature. The "prefreezeout" meson (especially kaon) evaporation during the phase transition is discussed in Sec. III. Section IV contains the calculation of the formation of the "strangelets" during the phase transition from quark-gluon plasma to hadron matter. Estimate of their size and charge-to-mass ratio are given. In the last section we show that the paircorrelation function of emitted $\Lambda$ 's can serve as a mean to detect the separation of strangeness during the phase transition; this correlation depends sensitively on the size of the $\Lambda$ 's location at freezeout.

\section{COLD STRANGE-QUARK MATTER}

Let us first discuss the properties of cold strangelets. If strange quarks are present, the Fermi energy of cold quark matter is lowered, provided the assumed current mass of the strange quark is smaller than the chemical potential $\mu_{q}$ of $u$ and $d$ quarks. Then the system is either metastable ${ }^{1,6}$ or even absolutely stable. ${ }^{2,3}$ A necessary condition for the stability of strange-quark matter against strong decay is that its energy per baryon must be smaller than that of the corresponding hyperonic matter. In Fig. 
1 we show the ground-state energy per baryon (i.e., at temperature $T=0$ and pressure $P=0$ ) as a function of the strangeness fraction $f_{s}$ (=net number of strangeness per baryon) as calculated in the MIT bag model ${ }^{3,6}$ for different values of the bag constant and strange-quark mass. In the present calculation quark matter is simply treated as a gas of noninteracting particles in a bag, its energy per baryon is then given by ${ }^{6}$

$$
\begin{array}{r}
E / A=B / n+n^{-1} \frac{3}{4 \pi^{2}}\left\{\left(\pi^{2} n_{u}\right)^{4 / 3}+\left(\pi^{2} n_{d}\right)^{4 / 3}+\frac{1}{2}\left[\left(\pi^{4 / 3} n_{s}^{2 / 3}+m_{s}^{2}\right)^{1 / 2}\left(\pi^{2} n_{s}\right)^{1 / 3}\left[2\left(\pi^{2} n_{s}\right)^{2 / 3}+m_{s}^{2}\right]\right.\right. \\
\left.\left.-m_{s}^{4} \ln \left[\frac{\left(\pi^{4 / 2} n_{s}^{2 / 3}+m_{s}^{2}\right)^{1 / 2}+\pi^{2 / 3} n_{s}^{1 / 3}}{m_{s}}\right]\right]\right\},
\end{array}
$$

where $n$ defines the baryon density, $n_{s}=f_{s} n$ defines the density of strange quarks, and where we neglected the mass of the up and down quark. The curves of Fig. 1 are calculated by assuming isospin symmetry $\left(n_{u}=n_{d}\right)$ and by minimizing the above expression for $E / A$ with respect to the density to describe the system at zero pressure. This ground-state energy of quark matter with fixed strangeness fraction $f_{s}$ is compared to the corresponding hadronic ground-state energy (dashed-dotted curve), determined as

$$
m_{\text {hyp }}=\left\{\begin{array}{l}
f_{s} m_{\Lambda}+\left(1-f_{s}\right) m_{N}-\epsilon_{B}, \quad 0 \leq f_{s} \leq 1, \\
\left(f_{s}-1\right) m_{\Xi}+\left(2-f_{s}\right) m_{\Lambda}-\epsilon_{B}, \quad 1 \leq f_{s} \leq 2, \\
\left(f_{s}-2\right) m_{\Omega}+\left(3-f_{s}\right) m_{\Xi}-\epsilon_{B}, \quad 2 \leq f_{s} \leq 3,
\end{array}\right.
$$

where $\Lambda, \Xi$, and $\Omega$ are the strange hyperons and $\epsilon_{B}$ is the binding energy per nucleon, which is, for simplicity, taken to be the infinite-nuclear-matter parameter of $15 \mathrm{MeV}$. For bag parameters $B^{1 / 4}$ lower than $190 \mathrm{MeV}$, strange-

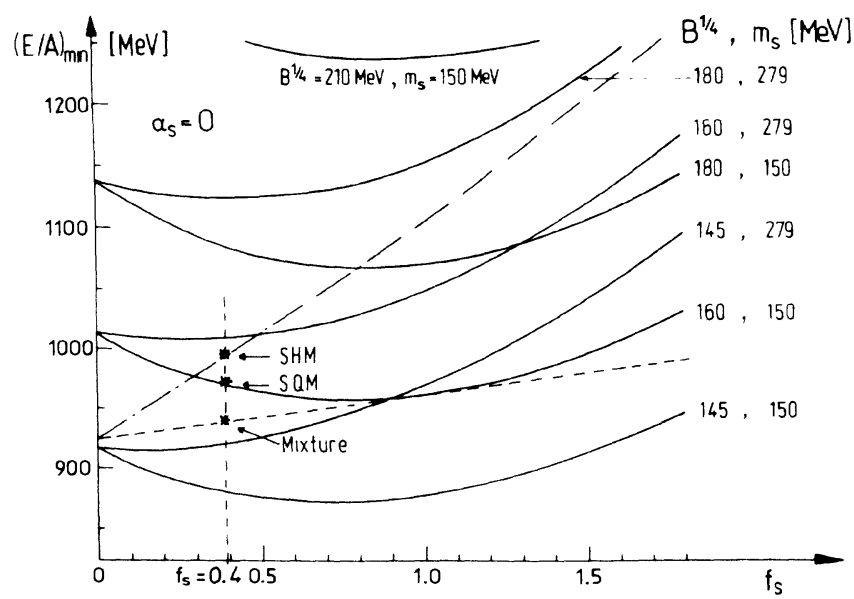

FIG. 1. Energy per baryon for strange-quark matter at zero temperature and zero pressure, as a function of the strangeness fraction $f_{s}\left[f_{s}=\left(\rho_{s}-\rho_{\bar{s}}\right) / \rho_{B} \equiv \rho_{s} / \rho_{B}\right.$ for zero temperature $]$ for different bag constants and strange masses. The dashed line defines the corresponding mass of a hyperonic matter ground state. A tangent construction shows that a mixture of both phases may be the true ground state because of lowest-energy per baryon. quark droplets can only decay via weak interactions, i.e., they are metastable. For larger $B$ values strangelets are unstable.

To investigate the importance of finite-size effects, the energy per baryon of finite strange-quark matter in a spherical MIT bag is calculated by filling up the bag with exact single-particle Dirac states, again assuming that the quarks do not interact. One has to solve the Dirac equation with a Bogolyubov-type boundary condition ${ }^{7}$ to describe the single-particle states:

$$
j_{l_{\kappa}}(p R)=-\operatorname{sgn}(\kappa) \frac{p}{E+m_{i}} j_{l_{-\kappa}}(p R)
$$

with

$$
\begin{aligned}
& p=\frac{\omega_{\kappa, \alpha}^{i}}{R}, \\
& E_{\kappa, \alpha}^{i}=\left[\left(\frac{\omega_{\kappa, \alpha}^{i}}{R}\right)^{2}+m_{i}^{2}\right]^{1 / 2} .
\end{aligned}
$$

Here $m_{i}$ is the quark mass $(i=u, d, s)$, with $m_{u}=m_{d}=0$ $\mathrm{MeV}$ and $m_{s}=150 \mathrm{MeV} . R$ defines the radius of the bag, $\kappa$ is the angular momentum quantum number, and $\alpha$ labels the eigenvalues in this quantum state $\kappa$. The total energy of the finite system is obtained by summing the "lowest"-energy eigenvalues and adding the phenomenological bag energy $B V$ for the perturbation vacuum in order to restore the discontinuity of the energy-momentum tensors at the surface of the quark bag. The volume $V$ or the radius $R$, respectively, has to be chosen in a way that the total pressure of the quarks inside the bag compensates the vacuum pressure $B$. We have included a center-of-momentum correction and a Coulomb term $\left[\left(N_{0}-2 N_{s}\right) / R\right]^{2} / 60 \alpha$, where $N_{0}$ is the number of massless up and down quarks and $N_{s}$ is the number of massive strange quarks. For details see Refs. 3 and 7.

One obvious difficulty is the fact that the momenta of the massive strange quarks do not scale with $1 / R$ for the above boundary problem. Hence one has to calculate a new set of energy eigenvalues for each value $R$. Pressure equilibrium is achieved by minimizing the total energy with respect to the radius. The results are depicted in Fig. 2.

Figure 2(a) shows the dependence of the energy per baryon of a giant bag on the baryon number for 
Bag with massless quarks

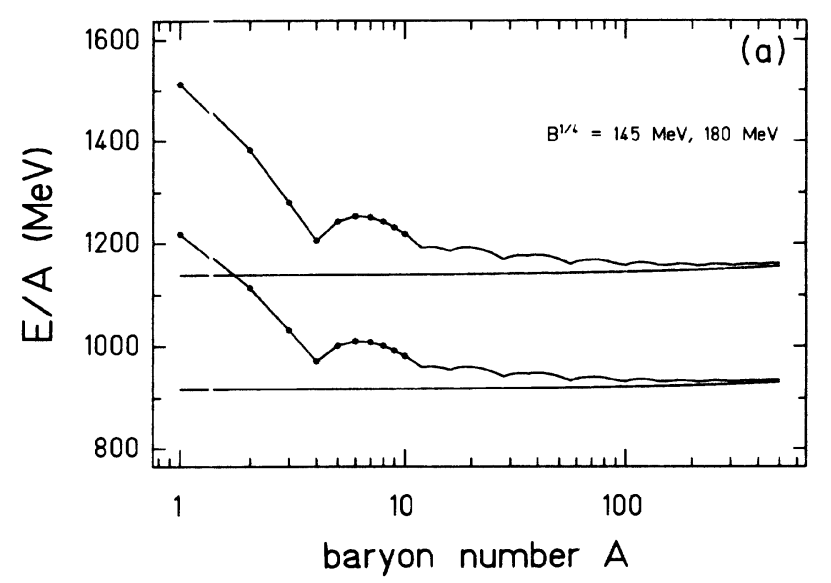

Bag with massive quarks

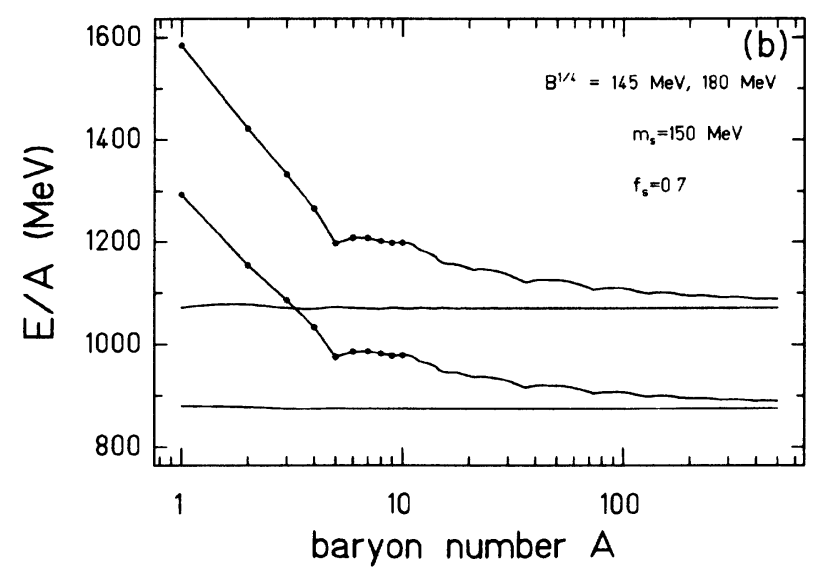

$\mathrm{B}^{1 / 4}=145 \mathrm{MeV}, \mathrm{m}_{\mathrm{s}}=150 \mathrm{MeV}$

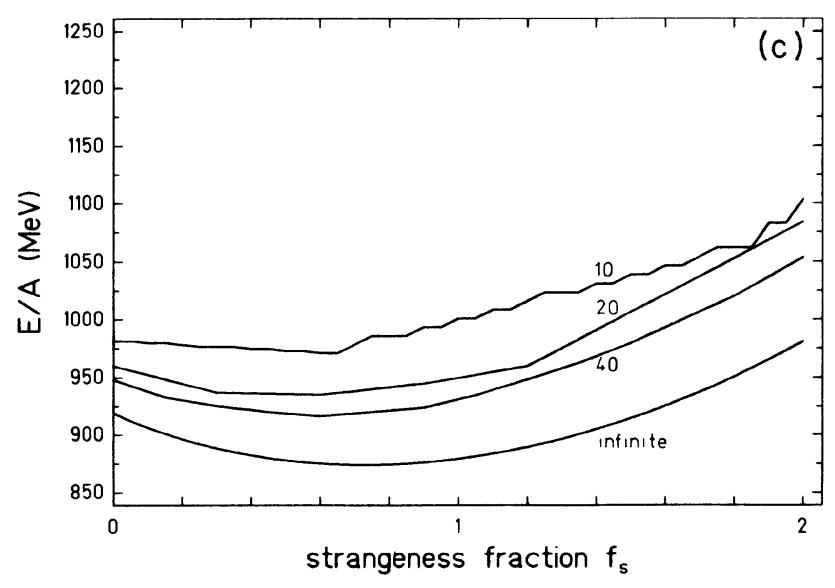

FIG. 2. $E / A$ vs $A$ or $f_{s}$, respectively, in a hadronic bag model for noninteracting $u, d$, and $s$ quarks, including Coulomb energy and center-of-mass correction. (a) Finite nonstrangequark matter with $B^{1 / 4}=145 \mathrm{MeV}$. (b) Finite strange-quark matter with $f_{s}=0.7$ and $B^{1 / 4}=145 \mathrm{MeV}$. (c) Finite strangequark matter with given baryon number $N_{B}=10,20,40$ vs the amount of included strange quarks $\left(f_{s}\right)$. The lowest curve in each figure shows the energy for infinite quark matter, as in Fig. 1.
$B^{1 / 4}=145$ and $180 \mathrm{MeV}$, respectively, and for a (constant) strangeness fraction of $f_{s}=0$, i.e., for pure (massless) up- and down-quark matter. If $f_{s}$ is set to 0.7 [Fig. 2 (b)], the total energy is lowered by $\sim 40 \mathrm{MeV} /$ nucleon, i.e., bags of up-, down-, and strange-quark matter are energetically favored compared to nonstrange quark bags. Note, however, that strange-quark matter is absolutely stable (as compared to nucleons) only for $B^{1 / 4}=145 \mathrm{MeV}$ (Ref. 3). The lower smooth curves in both figures are calculated in the thermodynamical limit, i.e., for infinite matter. One recognizes the shell effects in these figures. For very small bags $\left(N_{B}<10\right)$ the energy is at least 50 $\mathrm{MeV} /$ nucleon above the thermodynamical limit.

In Fig. 2(c) we regard the same quantities as in Fig. 1, but for finite baryon numbers $N_{B}=10,20,40$, varying the strangeness fraction of the bulk. Here we consider the bag value $B^{1 / 4}=145 \mathrm{MeV}$ only. The energy per baryon for these small $N_{B}$ values is about $50-100 \mathrm{MeV} /$ nucleon larger as compared to the infinite-quark-matter calculations (lowest curve). This can be interpreted ${ }^{3}$ in terms of a surface correction.

As was already noted in Ref. 7, even non-strange-quark matter might be absolutely stable, depending on the MIT bag constant [cf. Fig. 2(a)], but only for baryon numbers $N_{B}>300$.

Since the uncertainty due to the bag constant is much larger than the finite-size effects, we will use a thermodynamical description of the quark-matter droplets in the following (and also of hadronic matter for the same reasons).

Let us now return to the basic mechanism for the separation of strangeness, which leads to the formation of strange-quark-matter droplets in heavy-ion collisions (Sec. IV). This idea is demonstrated in a conspicuous manner (at $T=0$ ) in Fig. 1: Take a bag constant of 160 $\mathrm{MeV}$ and a strange-quark mass of $150 \mathrm{MeV}$. Consider a totally equilibrated system of cold nuclear matter in the ground-state with a finite total strangeness of 0.4 (see Fig. 1). One would now naively argue that strange-quark matter with $f_{s}=0.4$ is the ground state of the system, because the energy per baryon of this state is lower than that of the hyperonic state. However, the total energy per baryon can be lowered by an additional $\sim 50 \mathrm{MeV}$ by assembling the nonstrange quarks into pure nucleonic degrees of freedom, leaving the strange quarks in a strangematter droplet, its strangeness fraction enriched to $f_{s} \approx 1$. The minimal total energy is obtained with the tangent construction used in Fig. 1. Now the system consists of two phases, $60 \%$ of the baryon number contained in nucleons and $40 \%$ forming a strange-quark-matter cluster with $f_{s} \approx 1$. Hence we conclude that for $0<f_{s}<1$ the "true" ground state is a mixture of two phases, pure nucleonic matter and strange-quark matter (only for $B^{1 / 4}=145 \mathrm{MeV}$ a pure quark phase is the true ground state, even for $f_{s}=0$ ).

This separation of strange from nonstrange quarks and the unexpected occurrence of two different phases in the ground state should be further investigated because of its interesting consequences which are described in detail in the next sections. Therefore we now examine the Gibbs equilibrium phase transition between strange-quark 
matter and hadronic matter at $T=0$. For an explicit description of the phase-transition model we refer to Sec. IV, but we already note here the necessity of including a Hagedorn correction factor to account for the finite eigenvolume of the hadrons; otherwise the hadronic phase would be stable at large densities. ${ }^{8,9}$

The calculation shows that our first attempt to understand the physics of the strangeness separation by simple energetic arguments is indeed correct. If the system carries finite net strangeness, strange quarks will stay predominantly in the quark phase, thus leading to a mixture of two phases in the ground state (if we do not consider $B$ values for which strange-quark matter would be absolutely stable).
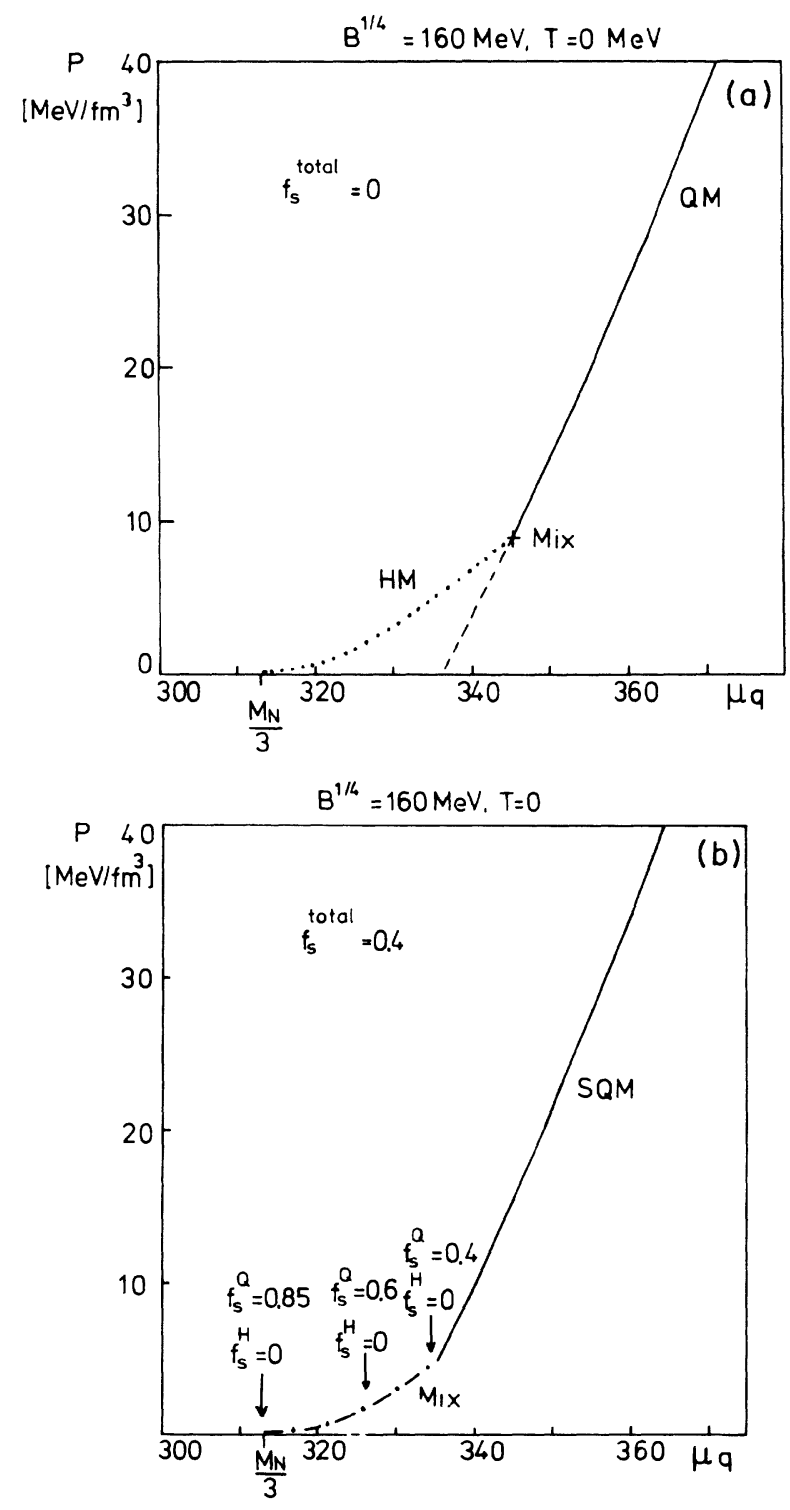

FIG. 3. Pressure vs baryochemical quark potential for the phase transition between quark and hadronic matter (a) with zero strangeness and (b) with finite strangeness $\left(f_{s}=0.4\right)$. One sees a principal difference between the two scenarios. In the case of finite strangeness, a "true" ground state has to be a mixture of both phases, a nonstrange hadronic, and a "strangelet."
In Figs. 3(a) and 3(b) this behavior is shown for $B^{1 / 4}=160 \mathrm{MeV}$ and for two values $f_{s}=0$ [Fig. 3(a)], 0.4 [Fig. 3(b)], where the pressure is depicted as a function of the quark chemical potential $\mu_{q}$. In Fig. 3(b) arrows indicate the strangeness content of each phase at various stages of the phase transition. As outlined above, there is a principal difference between these two situations: If there is no strangeness in the system, a phase transition occurs at one critical point $P_{\mathrm{cr}}\left(\mu_{q, \mathrm{cr}}\right)$, for smaller $\mu_{q}$ values down to $\mu_{q}=313 \mathrm{MeV} \sim M_{N} / 3$ the hadron phase is thermodynamically stable. However, if finite strangeness is considered, the phase transition starts at one critical $P_{\mathrm{cr}}\left(\mu_{q, \mathrm{cr}}\right)$, but continues until $P=0$, while all strangeness remains in the quark-matter phase. In our example a strangeness fraction of 0.85 and a fraction of baryon number of about 0.5 for the quark sector is obtained for the ground state, in perfect agreement with the tangent construction of Fig. 1, if one there neglects the global binding energy $\epsilon_{B}$.

This simple picture is also reflected in the finitetemperature calculations presented below. Indeed, this is the physical reason for the strangeness-separation mechanism in $f_{s}=0$ matter, which can lead to strangelet formation in high-energy heavy-ion collisions. ${ }^{5}$

\section{ENTROPY LOSS AND NET STRANGENESS ENRICHMENT DUE TO PION AND KAON EMISSION IN THE EXPANSION}

For the following arguments it is important to study the expansion of an initially hot quark-gluon-plasma fireball with special attention to the evolution of the strangeness during the phase transition (see Fig. 4). In Ref. 5 we have examined the fate of strange particles in an expanding system with zero net strangeness. However, a more realistic scenario must take into account the particle (i.e., mainly meson) radiation from the fireball before "freezeout." Let us make some crude estimates about the qualitative features of this radiation. First we have to consider meson radiation off the initial pure baryon-rich quark phase. One can think at least of seven different mechanisms: ${ }^{10-13}$ (a) a $q$ quark leaves the surface of the plasma, and due to $q \bar{q}$ or $s \bar{s}$ creation in a color-flux tube a $\pi$ or $K$ meson departs from the plasma; (b) as in (a), but with a $\bar{q}$ leaving the plasma, producing a $\pi$ or $\bar{K}$; (c) as in (a), but with an $s$ quark, producing a $\bar{K}$; (d) as in (a), but with an $\bar{s}$, producing a $K$; (e) a colorless, quasibound state of $q \bar{q}$ evaporates from the surface; (f) as in (e), but with $q \bar{s}$; and $(\mathrm{g})$ as in (e), but with $\bar{q} s$.

Anything else (such as $\phi$-meson radiation) can be regarded to be suppressed due to higher-mass thresholds. Qualitatively spoken, process (a) happens more often than (b) because of the overabundance of $q$ quarks $\left(\mu_{q}>0\right)$, while processes (c) and (d) happen with equal probability since $\mu_{s}=0$. Also, process (f) happens more often than (g), again since $\mu_{q}>0$. Hence, pions and $K^{+}, K^{0}$ are more easily radiated off the hot surface of the quark phase than $K^{-}$and $\bar{K}^{0}$ [see Fig. 4(a)]. Of course, in cases (a)-(d) the production rate of $K, \bar{K}$ depends not only on the chemical potential but also on the $s \bar{s}$ production rate in the flux tube. But, for the sake of simplicity, let us 


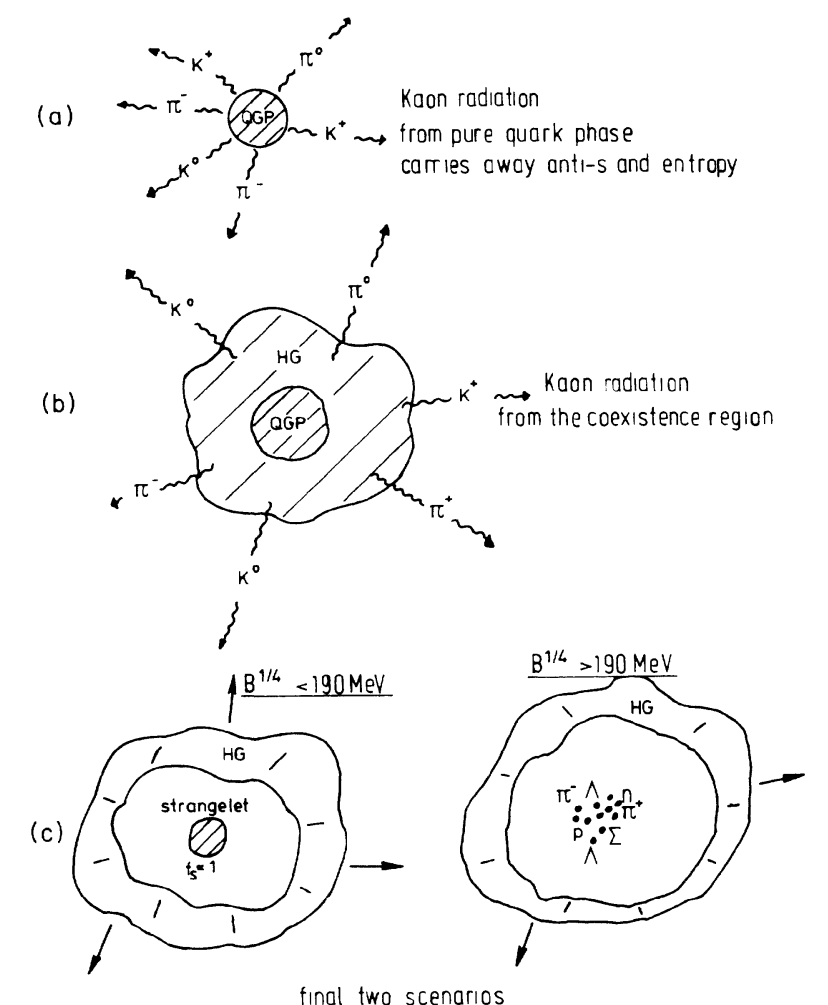

FIG. 4. Schematic picture of kaon $\left(K^{+}, K^{0}\right)$ radiation from a baryon-rich hot fireball (a) from a pure quark phase, (b) from the surrounding hadron phase after phase transition has started, (c) the two possibilities at the end of expansion, i.e., a "strangelet" is formed, which may be metastable, or multistrange hadrons are created in the last stage of the transition.

neglect this influence in the following calculation.

However, much more important than this process seems to be the meson radiation while the system is in the phase coexistence region. On one hand, most of the total collision time is spent in this region (see the results of the next section). On the other hand, the possibility of reabsorption of mesons is very unlikely, since the system approaches freezeout.

We have shown ${ }^{5}$ that during the phase coexistence the quark phase is charged up with strangeness leading to $f_{s} \leq 0.8 . K^{+}$and $K^{0}$ mesons, ${ }^{14}$ which absorb the $\bar{s}$ quarks in the hadronic sector, are enriched while the hyperons are suppressed. Pions and $K^{+}, K^{0}$ are lighter than the baryons, their thermal velocity is higher and therefore they are emitted more rapidly. Thus the system cools by thermal evaporation in addition to the cooling due to the isentropic expansion (see Fig. 5).

The meson evaporation in both stages of the expansion just described carries away entropy, energy, and antistrangeness. Therefore the residual expanding fireball, which is in the mixed phase, loses entropy and is charged up with net strangeness (see Fig. 4). We will show below that this additionally increases the strangeness abundance in the quark phase [see Figs. 5(a) and 10], even the value of $f_{s} \sim 0.5$ calculated in Ref. 5. Furthermore the entropy in the quark phase drops quite strongly, while the hadron phase dilutes immensely and hence absorbs the remaining entropy.

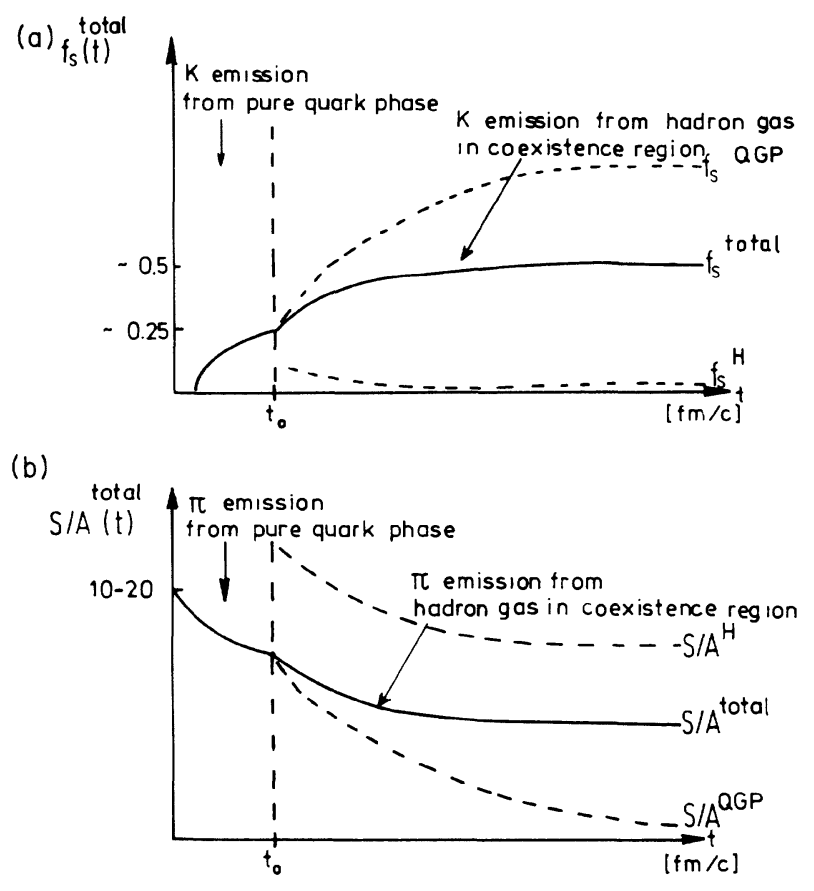

FIG. 5. An estimate of the enrichment of strangeness (a) and the loss in entropy (b) of the remaining system during expansion of the fireball.

To prime this picture, let us give a rough estimate of the entropy loss and the kaon emission during the expansion process of the fireball by a blackbody radiation formula: The pion and kaon densities in a thermalized system are

$$
\begin{aligned}
& \rho_{\pi}=\frac{3}{2 \pi^{2}} 2.4 T^{3}, \\
& \rho_{K}=2 \frac{T^{3}}{2 \pi^{2}}\left(\frac{\pi}{2}\right)^{1 / 2}\left(\frac{m_{K}}{T}\right)^{3 / 2} e^{-m_{K} / T} e^{\mu_{K} / T}, \\
& \rho_{\bar{K}}=\rho_{K} e^{-2 \mu_{K} / T} .
\end{aligned}
$$

Here $m_{K}=500 \mathrm{MeV}$ is the mass of the kaon and $\mu_{K}=\mu_{q}-\mu_{s}$ is its chemical potential. The antikaons are suppressed by a Boltzmann factor $e^{-2 \mu_{K} / T}$ as compared to the kaons. In the quark phase at least $\rho_{\pi} / 2$ pions and $\rho_{K} / 2$ kaons, respectively, leave a surface layer per unit time and area. This can be motivated, if we assume that the surface of the quark phase is covered by a thin, thermalized cloud of mesons, its temperature and chemical composition implied by the quark phase. At the relevant temperatures mesons have nearly the speed of light. Obviously half of the particles have the possibility to leave the cloud, while the other half are emitted back into the plasma. This agrees with the results of Ref. 13. However, during the phase coexistence nearly all $\rho_{\pi}$ pions and $\rho_{K}$ kaons, respectively, spatter off the outer surface of the hadron phase, because the outer layer of mesons moves with nearly the speed of light, rapidly losing the thermal contact of the rest of the remaining fireball.

Each pion carries $\sim 4$ units of entropy and each kaon carries one unit of antistrangeness; hence the loss of entropy per time is given by the size of the surface times 
TABLE I. Calculated properties of QGP and mixed phases.

\begin{tabular}{|c|c|c|c|c|c|c|c|c|}
\hline $\begin{array}{c}\text { Stage } \\
\text { of system }\end{array}$ & $\begin{array}{c}\text { Baryon } \\
\text { density } \rho \\
\left(\rho_{0}\right)\end{array}$ & $\begin{array}{c}\text { Temperature } \\
T \\
(\mathrm{MeV})\end{array}$ & $\begin{array}{c}\mu_{K} \\
(\mathrm{MeV})\end{array}$ & $\frac{d(S / A)}{d t}$ & $\begin{array}{c}\frac{d f_{s}}{d t} \\
(1 / \mathrm{fm})\end{array}$ & $\begin{array}{c}\Delta t \\
(\mathrm{fm})\end{array}$ & $\Delta \frac{S}{A}$ & $\Delta f_{s}$ \\
\hline QGP & 4 & 200 & 150 & -0.99 & 0.046 & 5 & -4.95 & 0.23 \\
\hline Mixed & 3 & 100 & 200 & -0.3 & 0.014 & 20 & -5.98 & 0.29 \\
\hline Mixed & 2 & 50 & 300 & -0.049 & 0.0025 & 100 & -4.9 & 0.25 \\
\hline
\end{tabular}

four times the outgoing pion density. The gain of strangeness is obtained analogously

$$
\begin{aligned}
& \frac{d(S / A)}{d t} \sim-\frac{1}{N_{B}} 4 \pi\left[\frac{3}{4 \pi} \frac{N_{B}}{\rho_{B}}\right]^{2 / 3} 4 \rho_{\pi} \times\left(1 \text { or } \frac{1}{2}\right), \\
& \frac{d f_{S}}{d t} \sim-\frac{1}{N_{B}} 4 \pi\left[\frac{3}{4 \pi} \frac{N_{B}}{\rho_{B}}\right]^{2 / 3} 4\left(\rho_{K}-\rho_{\bar{K}}\right) \times\left(1 \text { or } \frac{1}{2}\right) .
\end{aligned}
$$

The resulting rates are listed in Table I, assuming a total baryon number of $N_{B}=100$. The quantities $\rho_{B}, T, \mu_{K}$ are estimated from the calculations of the next section. These quantities stem, however, from a calculation assuming thermodynamical equilibrium and isentropic expansion. They should in principle be consistently calculated in a rate-equation approach to describe the nonequilibrium time evolution of the considered observables quantitatively. For the time being, we will be content with the qualitative picture developed here.

$\Delta t$ is the time the system stays in the specified stage, which is obtained from the later calculations assuming a longitudinal expansion of the hot fireball. We have examined three different stages of the fireball, first as a hot quark-gluon droplet, second at the early stage of the phase transition, and third at a later stage of the transition with lower temperature. Adding all numbers, a loss in entropy per baryon of about 15 and a strangeness enrichment of the total system to about $f_{s}=0.75$ is found.

Figures $5(\mathrm{a})$ and $5(\mathrm{~b})$ depict qualitatively the possible time dependence of the total strangeness $f_{s}(t)$ in the system and the total entropy, respectively.

Figure 4(c) describes the two alternatives for the final state of the reaction. As will become clear in the next section, for $B<190 \mathrm{MeV}$ the pion and kaon radiation leads to a mixed phase of quark droplets and hadrons in the late stage of the expansion. This mixture attains a lower energy per baryon than the pure hadron gas. Consequently the phase transition is brought to a halt. The system is left in a metastable state of two phases at low temperatures and at nearly zero pressure, containing the strangelet as survivor of the quark-gluon-plasma phase. On the other hand, for $B$ values $>190 \mathrm{MeV}$ strangequark matter will decay predominantly into hyperons. We will examine this possibility in the conclusions.

\section{NUMERICAL CALCULATION OF STRANGELET FORMATION}

Let us now investigate the consequences of the abovementioned nonequilibrium meson and kaon evaporation assuming a longitudinal expansion of the system: ${ }^{15}$

$$
\rho_{B}(t)=\rho_{B}\left(t_{0}\right) \frac{t_{0}}{t} \quad\left(t_{0} \simeq 5 \mathrm{fm} / c\right) .
$$

In an equilibrium expansion (without meson radiation) entropy and net strangeness would be conserved, but the actual expansion must, of course, follow a path along which $f_{s}^{\text {total }}$ is steadily increasing and the total entropy is steadily decreasing with time due to pion and kaon radiation (see Fig. 5).

A quantitative nonequilibrium (e.g., rate equation) analysis of the complete time-dependent microscopic evolution is beyond the scope of the present paper. Here we simply estimate the nonisentropic expansion of the system using various finite (i.e., increased), but constant net strangeness fractions $f_{s}>0$ and decreased entropies $S / A<S /\left.A\right|_{\text {initial }}$ to simulate the actual time-dependent increasing net strangeness and decreasing entropy values.

We employ the bag-model equation of state for the quark-gluon plasma ${ }^{9}$ and take hadron matter to be a mixture of relativistic Bose-Einstein and Fermi-Dirac gases of the well-established nonstrange and strange hadrons up to $M \simeq 2 \mathrm{GeV}$. Hadronic densities are corrected by a Hagedorn eigenvolume factor in order to take account for the repulsive interactions. ${ }^{8,9}$

When reaching the phase-coexistence region, defined by Gibbs's conditions $\left(T^{\mathrm{QGP}}=T^{\text {had }}, \quad P^{\mathrm{QGP}}=P^{\text {had }}\right.$, $\mu_{q}^{\mathrm{QGP}}=\mu_{q}^{\text {had }}, \mu_{s}^{\mathrm{QGP}}=\mu_{s}^{\text {had }}$ ), baryon number and entropy conservation force the system to expand along the phase boundary by converting plasma volume into hadron gas volume. ${ }^{5}$ For a given entropy and baryon number, the temperature and the baryochemical potential specify the volume occupied by the two phases. The strange chemical potential $\mu_{s}$ needs to be specified to ensure strangeness conservation. The following system of nonlinear equations is solved for $T, \mu_{q}, \mu_{s}$ :

$$
\begin{aligned}
& P^{\mathrm{QGP}}\left(\mu_{q}, \mu_{s}, T\right)=P^{\mathrm{had}}\left(\mu_{q}, \mu_{s}, T\right), \\
& f_{s}=f_{s}^{\mathrm{QGP}}\left(\mu_{q}, \mu_{s}, T\right) \lambda_{B}^{\mathrm{QGP}}+f_{s}^{\mathrm{had}}\left(\mu_{q}, \mu_{s}, T\right)\left(1-\lambda_{B}^{\mathrm{QGP}}\right), \\
& \frac{S}{A}=\left[\frac{S}{A}\right]^{\mathrm{QGP}}\left(\mu_{q}, \mu_{s}, T\right) \lambda_{B}^{\mathrm{QGP}} \\
& \quad+\left[\frac{S}{A}\right]^{\text {had }}\left(\mu_{q}, \mu_{s}, T\right)\left(1-\lambda_{B}^{\mathrm{QGP}}\right),
\end{aligned}
$$

where $S / A$ and $f_{S}$ are constants. In addition, $\lambda_{B}^{\text {QGP }}=N_{B}^{\text {QGP }} / N_{B}^{\text {total }}$, i.e., the fraction of baryons in the quark phase, has to be specified to obtain a unique solution of the above equations. Obviously $\lambda_{B}^{\mathrm{QGP}}$ is decreasing with time while hadronization proceeds. 


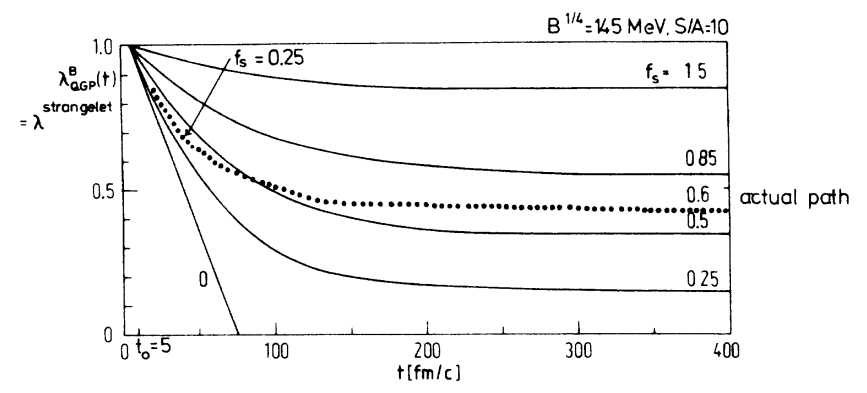

FIG. 6. Time evolution of the amount of baryon number $\left[\lambda_{B}^{\mathrm{OGP}}(t)=N_{B}^{\mathrm{QGP}} / N_{B}^{\text {total }}\right]$ in the remaining quark phase. Isentropic, one-dimensional expansion with $S / A=10$, starting point of the transition is $t_{0}=5 \mathrm{fm} / c$, with certain initial strangeness fraction $f_{s}\left(t_{0}\right)=f_{s}=$ const. Note that only for $f_{s}=0$ the system totally converts into hadrons. $B^{1 / 4}=145 \mathrm{MeV}, m_{s}=150 \mathrm{MeV}$, $\rho_{B}\left(t_{0}\right) / \rho_{B}(t)=t / t_{0}, t \geq t_{0}=5 \mathrm{fm} / c$.

We constrain ourselves to use the original MIT bag value $B^{1 / 4}=145 \mathrm{MeV}$ and $m_{s}=150 \mathrm{MeV}$ throughout the results presented below. The influence of the bag constant will be discussed later for one example. Figure 6 shows the fraction $\lambda_{B}^{\text {QGP }}=N_{B}^{\text {QGP }} / N_{B}^{\text {total }}$ of baryons in the quark phase as a function of time for an entropy per baryon of 10 for different net strangeness fractions $f_{s}$ of the total system. We remark that for zero total strangeness $\left[f_{s}\left(t_{0}\right)=0\right]$ the quark phase totally converts into hadrons. However, the prefreezeout kaon radiation discussed above forces the system from an initial net strangeness of zero to finite $f_{s}(t)$ values. We see that even for small $f_{s}$ values $(\simeq 0.25)$ the conversion from quark matter into hadrons comes to a halt in a prolonged phase transition at a point where the pressure of the system approaches zero. We point out that the resulting long expansion time supports our assumption that the time scale of chemical transmutations is short as compared to the system's lifetime. It also emphasizes the importance of the emission of particles from the outer layers of the system.

Very important is the enhancement in $f_{s}$ of the total system due to kaon radiation which leads to even larger $\lambda_{B}^{\text {OGP }}$ values at a given entropy (neglecting for a moment that the total entropy has to decrease). This is illustrated in Fig. 6 by the actual path (dotted line), which indicates the increase of $f_{s}$ with time.

As we will demonstrate in the following figures, the remaining quark droplet has the properties of a "strangelet" described above. To get an estimate of its size, let us assume a total initial baryon number of 100 . The number of baryons in the droplet is then given by $N_{B}^{\text {droplet }}=\lambda_{B}^{\mathrm{QGP}}(t \rightarrow \infty) \times 100$ and is typically in the range of $40 \pm 20$ (cf. Fig. 6).

In Fig. 7(a) we fix the total net strangeness at $f_{s}=0.25$ and show the evolution of the baryon number in the quark droplet $\lambda_{B}^{\text {QGP }}$ as a function of time for various entropy per baryon ratios ranging from 3 to 25 . Note that only for values $S / A \leq 20$ the phase transition actually comes to a halt. Then strangelets are formed, which have masses in the range from 60 to 5 for $S / A=3$ to $S / A=15$. However, the initial entropy must have been considerably larger than these $S / A$ values, because of the loss of entropy due to the meson emission discussed above. A qualitative estimate of the time dependence of $(S / A)(t)$, i.e., of the actual path followed by the system, is indicated by the dotted line (neglecting for the moment the increase in $f_{s}$ ).

Qualitatively similar results for bag constants $B^{1 / 4}=160$ and $180 \mathrm{MeV}$ are depicted in Figs. $7(\mathrm{~b})$ and 7 (c). However, as one would guess from the detailed investigations of strange-quark matter at zero temperatures in Sec. II, this strangelet formation can in principle only work for bag constants smaller than $190 \mathrm{MeV}$; for $B>190 \mathrm{MeV}$ the quark phase always converts totally into hadrons because of its unfavorably high energy (as shown in Sec. II). Also, using a bag constant close to 190 $\mathrm{MeV}, S / A$ has to be small (in our examples $S / A \leq 7.5$ ) for strangelet formation to be feasible. Higher entropy
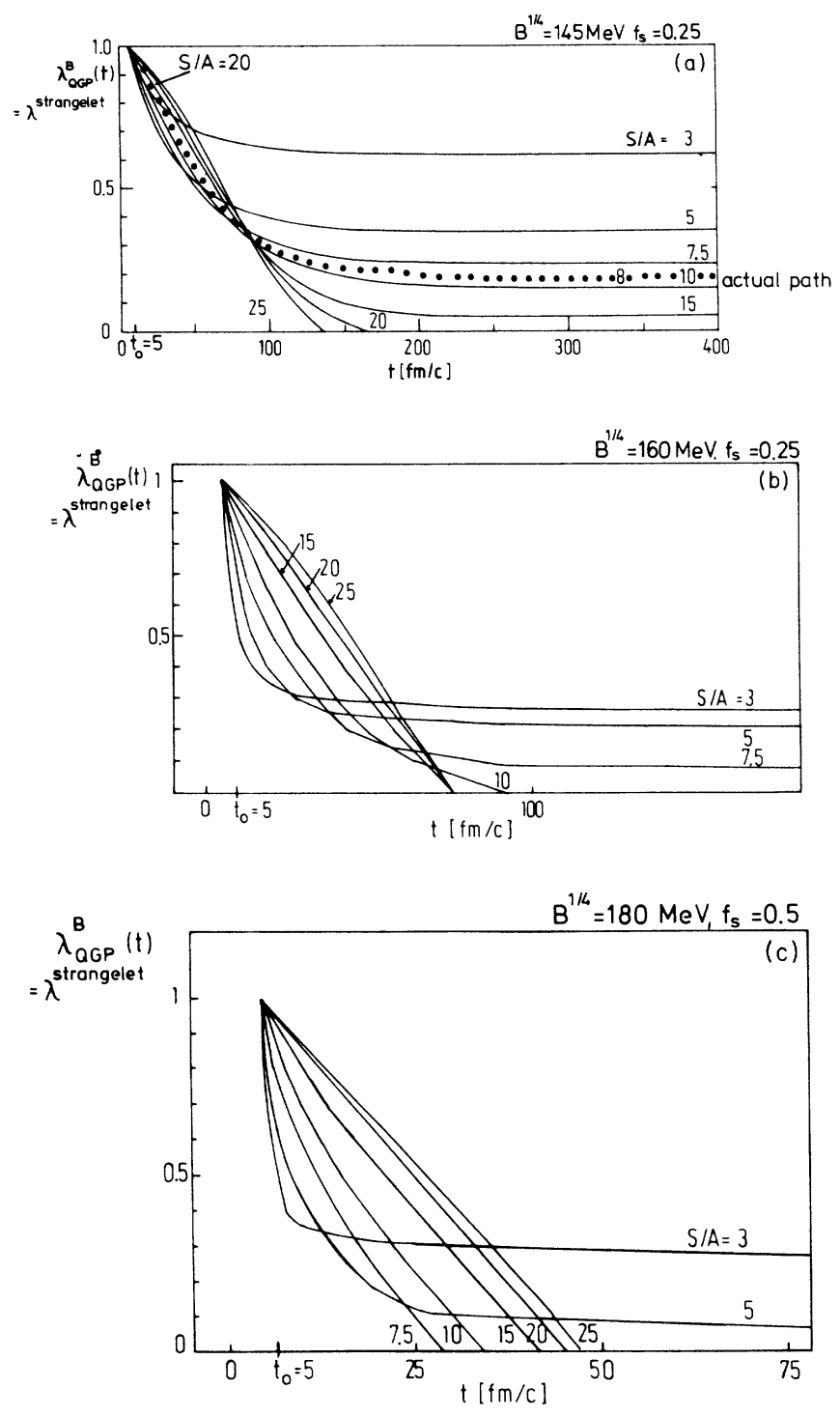

FIG. 7. (a) The same as in Fig. 6, but for fixed $f_{s}\left(t_{0}\right)=f_{s}=0.25$ and varied entropy per baryon. Note that only for $S / A=20,25$ the system converts totally into hadrons. (b) The same as in (a), but for $B^{1 / 4}=160 \mathrm{MeV}$. (c) The same as in (a), but for $B^{1 / 4}=180 \mathrm{MeV}$ and $f_{s}\left(t_{0}\right)=f_{s}=0.5$. 


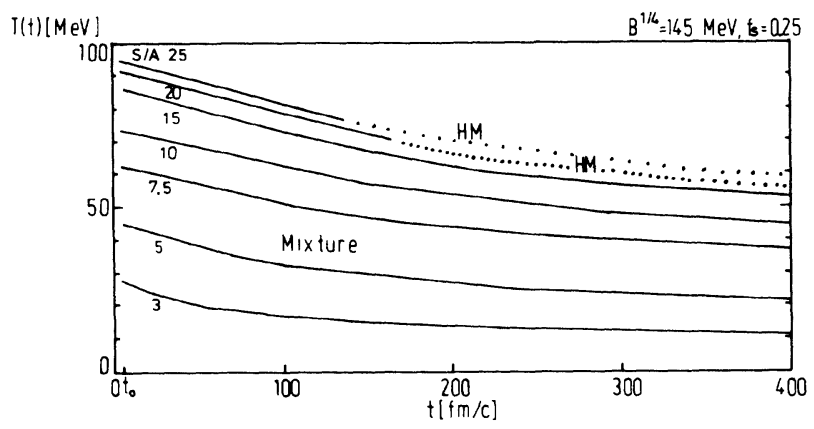

FIG. 8. The time evolution for the temperature during isentropic expansion. One observes a large decrease in temperature. The conditions are the same as in Fig. 7(a).

means higher temperature, and because the quark phase obtains more thermal degrees of freedom, its energy rises more rapidly with temperature than the hadron resonance gas. The energy of the quark phase rises linearly with the bag constant. Thus, if $B$ is larger, the quark phase becomes unstable at such temperatures, where it would be stable for smaller bag constants.

Eventually the adiabatic expansion has cooled the total system, due to diluting hadrons, as depicted in Fig. 8, where the time evolution of temperature is shown for a strangeness fraction $f_{s}=0.25$ and different entropy per baryon ratios. The entropy per baryon of the quarkmatter phase decreases dramatically, because of its finite density which lies around two times the density of normal nuclear matter. Nearly all entropy is absorbed by the surrounding dilute hadron gas, mainly nucleons and pions. This entropy splitting between both phases during the phase transition, already discussed in Fig. 5, is qualitatively shown for $S / A=5,15,25$ in Fig. 9. One clearly recognizes the large decrease of the entropy of the quark-matter droplet. At some critical point thermal contact cannot be further maintained, freezeout happens, thus leaving a rather cold strangelet.

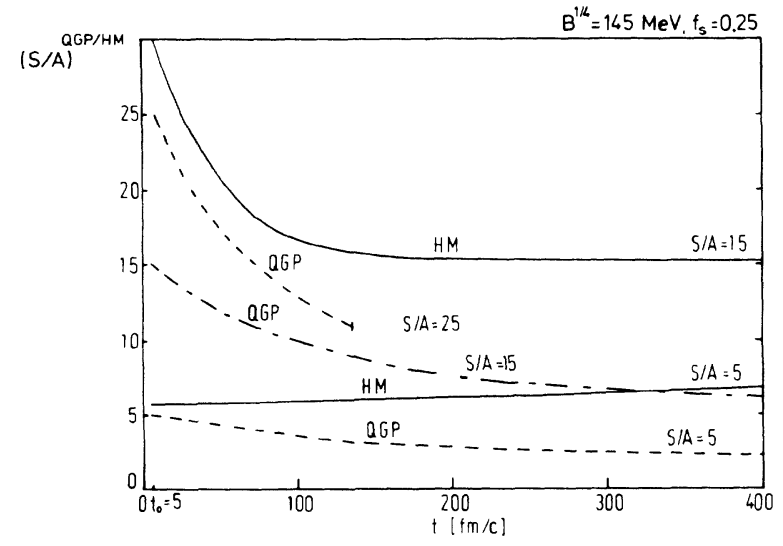

FIG. 9. The time evolution of the entropy in the two individual phases while passing through the coexistence region. Note that the entropy per baryon drops drastically in the strangelet, and the surrounding hadron phase absorbs the entropy of the system. The conditions are the same as in Fig. 7(a).

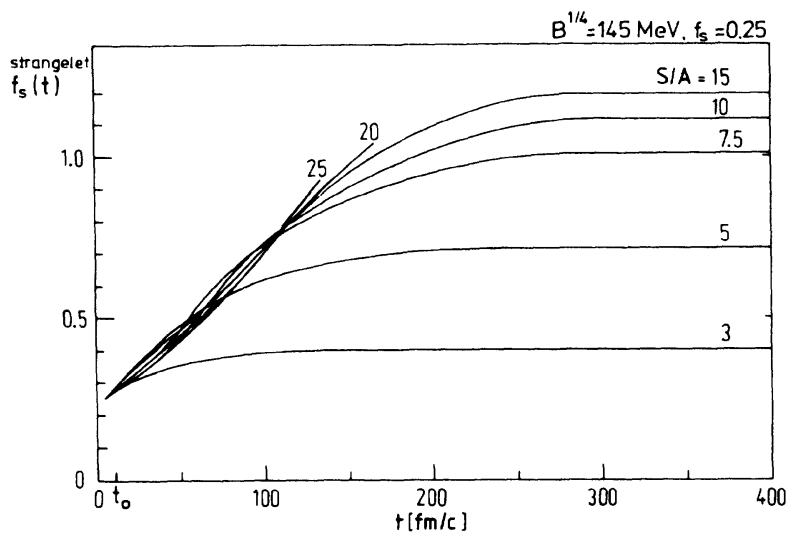

FIG. 10. The time evolution of the fraction of strangeness in the strangelet. One observes a strong enrichment of $f_{s}$ with time, all strange quarks remain more or less in the quark droplet. The conditions are the same as in Fig. 7(a).

We now want to describe some of the properties of this remaining strangelet, which in fact are similar to those of the cold $(T=0)$ strangelet described in Sec. II. Figure 10 depicts the amount of strangeness in the strange-quarkmatter droplet calculated for the same parameters as in Fig. 7(b). A drastic enrichment of $f_{s}^{\text {droplet }}$ can be seen. In addition be aware of the decreasing total entropy and increasing total strangeness fraction of the system due to particle evaporation. Typical $f_{s}$ values of the final droplet lie around 0.8. Figure 11 depicts the development of baryon density of the created strangelet, the final values are about 1.5-2 of normal nuclear density, which agrees with the values for cold matter. The droplets tend to drift towards the minimum energy per baryon on the curves shown in Fig. 1 by additional emission of nucleons, and also pions, antikaons, and photons.

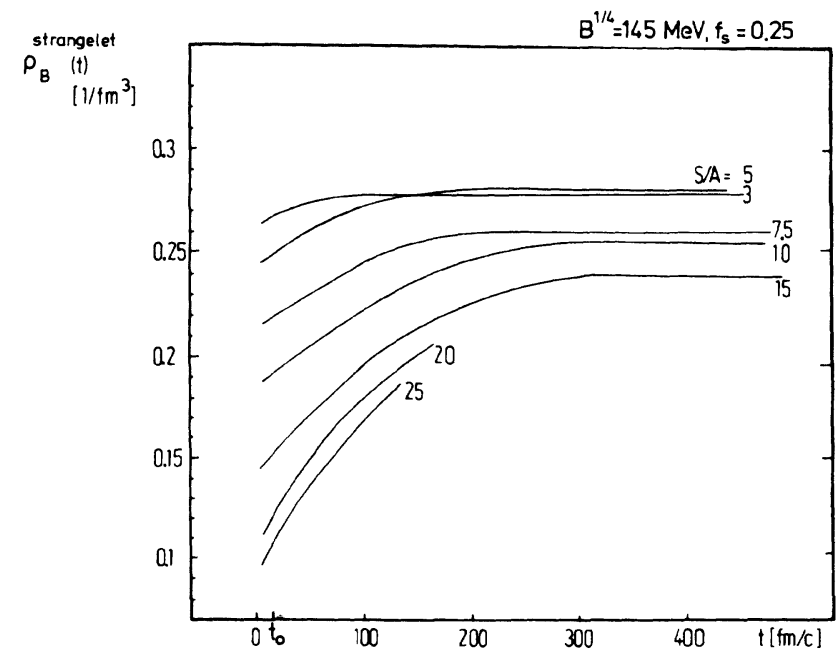

FIG. 11. The time evolution of the baryon density of the strange-quark blob. Note that the density of the strangelet lies around 1.5-2 of normal nuclear matter. The conditions are the same as in Fig. 7(a). 
Hence we summarize that kaon and pion radiation in fact favors the creation of relatively cold, nearly neutral, multi-strange-quark-matter droplets, their mass can be as large as $50 \%$ of the total mass of the system, their charge-to-mass ratio $Z / A \leq 0.3$, their density around two times the ground-state density of nuclear matter.

\section{OUTLOOK: DETECTING STRANGELETS}

We conclude that there are two processes which would suggest the formation of "strangelets" in ultrarelativistic heavy-ion collisions, if a baryon-rich quark-gluon plasma is formed. The first is due to the $s-\bar{s}$ separation mechanism presented earlier. ${ }^{5}$ This leaves us with a quark phase in the coexistence region of hadronic and quark matter, which is charged up with strangeness. The $s$ quarks, created in pairs with $\bar{s}$ quarks in the early quarkgluon fireball, remain in the quark phase during the phase transition, the $\bar{s}$ quarks materialize mainly into kaons.

Second, the evaporation of pions and kaons from the system, in particular during the phase transition, cools the matter and charges it up with net strangeness. Thus, because of $f_{s}^{\text {total }}>0$, the chemical equilibrium composition of strangeness is shifted towards additional strange quarks, which also get trapped in the quark phase. Now, while the phase transition proceeds, the system cools and, similar to strange-quark matter at zero temperature (Sec. II), has its smallest energy per baryon number for a mixture of nucleons and a droplet of strange-quark matter. When the thermal freezeout stage is reached, a cold strange-quark-matter droplet is left. This is in contrast with Ref. 16, where it was claimed that our original proposal might never work because the system would have to decay due to its large entropy content. However, entropy and heat are carried away by evaporated pions and nucleons. Thus these strangelets might survive for times $t>10^{-4} \mathrm{sec}$ after collision, even if these objects were only metastable and could decay solely via weak interactions.

A microscopic nonequilibrium calculation of the time evolution of the quark-gluon plasma is urgently needed to quantify the simple estimates presented above.

Other questions, which have not yet been tackled, are as follows. (a) At what bombarding energy would strangelets be formed? (b) What are the corresponding entropy per baryon values? (c) How could a strangelet be detected experimentally?

In Fig. 12 the entropy per baryon is shown as calculated in a one-dimensional hydrodynamical model ${ }^{15,17}$ as a function of $E_{\mathrm{lab}}^{\mathrm{kin}}$ for a gas of hadronic resonances and deconfined plasma with $B^{1 / 4}=180 \mathrm{MeV}$. For details of this calculation see Refs. 15 and 17. One observes that according to these calculations a quark-gluon plasma could be created at rather moderate bombarding energies. For bombarding energies $E_{\text {lab }}^{\text {kin }}=10-50 \mathrm{GeV} /$ nucleon the resulting low entropy per baryon values would favor strangelet formation. In collision with bombarding energies of $200 \mathrm{GeV} /$ nucleon the entropy in the target fragmentation regions would also be moderate enough for quark-matter formation and thus for strangelet creation.

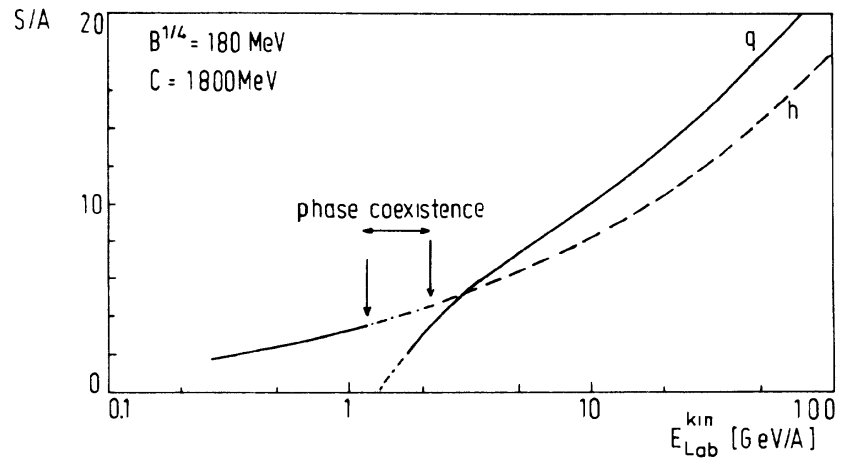

FIG. 12. The entropy produced in a hydrodynamical model is shown as a function of $E_{\mathrm{lab}}^{\mathrm{kin}}$ for nuclear matter and deconfined plasma phase with a bag constant of $180 \mathrm{MeV}$.

If a strangelet were actually produced in a heavy-ion collision, it would be the only unique signal for the formation of a quark-gluon plasma. It could be detected by its unusually small charge-to-mass ratio. It would thus appear similar to, e.g., a proton with unusually large transverse momentum. A strangelet can be detected by interacting with a secondary nucleus: multiple production of $\Lambda$ 's in such a secondary reaction would serve as a signal for the strange-quark-matter droplet. A neutral strangelet can be observed in calorimeters by its large total energy and transverse mass. (Weak) decay in flight could be observed. We note that the decay modes of strangelets have been investigated by Chin and Kerman. ${ }^{6}$

If the bag constant were larger than $190 \mathrm{MeV}$, strange-quark matter would be unstable even for zero temperature (Fig. 1). However, even then the quark phase becomes strongly enriched with strange quarks during the phase transition. This behavior is shown in Fig. 13(a), where $f_{s}^{\mathrm{QGP}}$ in the phase coexistence region is depicted as a function of $\mu_{q}$ for various values of the volume fraction $\chi=V_{\text {had }} /\left(V_{\mathrm{QGP}}+V_{\text {had }}\right)$ of the hadronic phase $\left(f_{s}^{\text {total }}=0.25\right.$ and $\left.B^{1 / 4}=210 \mathrm{MeV}\right)$. We remark that even for $T \rightarrow 0$ (or equivalently, for large $\mu_{q}$ ) $f_{s}^{\mathrm{QGP}}$ does not converge towards zero (as would be the case for $f_{s}^{\text {total }}=0$, since almost no $\bar{s}$ quarks and no $s$ quarks can be produced at low temperatures, cf. Ref. 5). The $\Lambda$ 's would emerge at the very end of the hadronization phase transition, as demonstrated in Fig. 13(b), where the increase of the number of $\Lambda$ 's per unit change of the baryon-number fraction $\lambda_{B}^{\text {had }}$ of the hadronic phase, $d N_{\Lambda} / d \lambda_{B}^{\text {had }}$, is depicted as a function of $\lambda_{B}^{\text {had }}=1-\lambda_{B}^{Q G P}$, assuming a total baryon number of $N_{B}^{\text {total }}=100$ and an entropy per baryon ratio of 15 . Since then the quark-phase volume is rather small, the $\Lambda$ 's would be strongly localized in space-time. Strong localization implies that two produced $\Lambda$ 's should be strongly correlated. If deconfinement does not occur in a collision, i.e., if a highly compressed, thermalized gas of hadrons is formed, the $\Lambda$ 's are created over a much larger region in space-time, namely, throughout the whole fireball. The two-particle correlation function has then a different shape.

The function $R\left(\mathbf{p}_{\text {rel }}\right)$ (where $\mathbf{p}_{\text {rel }}$ is the relative momentum of the two particles) can be obtained by an ansatz 


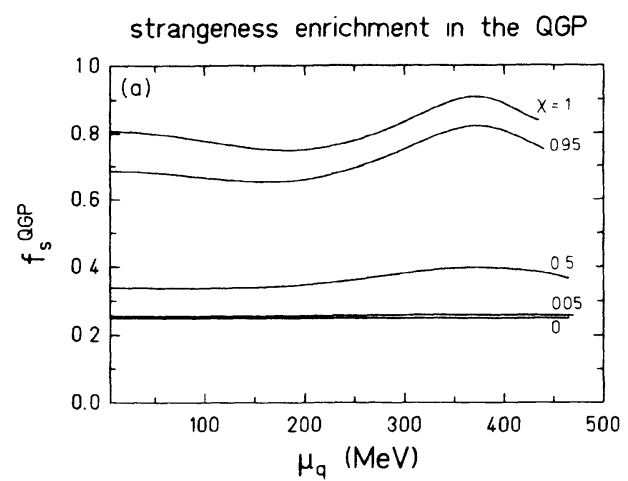

$B^{t / 6}=210 \mathrm{MeV}$ $m_{s}=150 \mathrm{MeV}$ $t_{s}=025$ Lambda emission out of the QGP

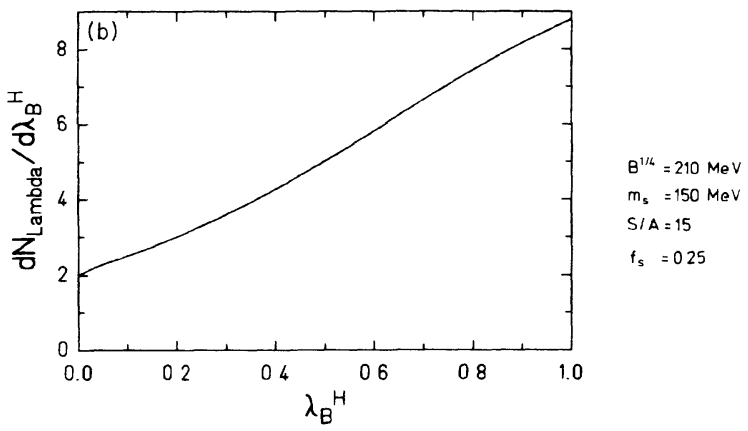

FIG. 13. (a) $f_{\mathrm{s}}^{\mathrm{QGP}}$ as a function of $\mu_{q}$ for various volume fraction $\chi=V_{\text {had }} /\left(V_{\mathrm{QGP}}+V_{\text {had }}\right), B^{1 / 4}=210 \mathrm{MeV}, f_{s}^{\text {total }}=0.25$. (b) Production of $\Lambda$ 's increases towards the end of the phase transition for an assumed entropy to baryon ratio of 15 [the other parameters are the same as in (a)].

similar to the one used earlier for $p$ - $p$ (Hanbury-BrownTwiss) correlations: ${ }^{18}$

$$
\begin{aligned}
& \frac{1}{\sigma_{12}} \frac{d^{2} \sigma_{12}}{d \mathbf{p}_{1} d \mathbf{p}_{2}} \simeq\left[1+R\left(\mathbf{p}_{\text {rel }}\right)\right] \frac{1}{\sigma_{1}} \frac{d \sigma_{1}}{d \mathbf{p}_{1}} \frac{1}{\sigma_{2}} \frac{d \sigma_{2}}{d \mathbf{p}_{2}}, \\
& \mathbf{p}_{1} \approx \mathbf{p}_{2} \approx \mathbf{p}_{\text {c. } \mathrm{m} .},
\end{aligned}
$$

where $\mathbf{p}_{1}, \mathbf{p}_{2}$ are the momenta of the two particles and $\mathbf{p}_{\text {c.m. }}$ and $\mathbf{p}_{\text {rel }}$ describe the center of mass and relative momenta, respectively. $R$ ( $\left.\mathbf{p}_{\text {rel }}\right)$ defines the difference between the differential two-particle cross section and the product of the inclusive single-particle cross sections. $R$ should only depend on the size $r_{0}$ of the area where the particles are located at freezeout and from where they are emitted. Considering the spin distribution to be statistical and introducing a spatial distribution

$$
D(r, \mathbf{p})=\frac{1}{\sigma_{1}} \frac{d \sigma_{1}}{d \mathbf{p}}\left(e^{-r^{2} / r_{0}^{2}} / \pi^{3 / 2} r_{0}^{3}\right)
$$

for the emitted wave packets, one finds
Lambda correlation without resonance

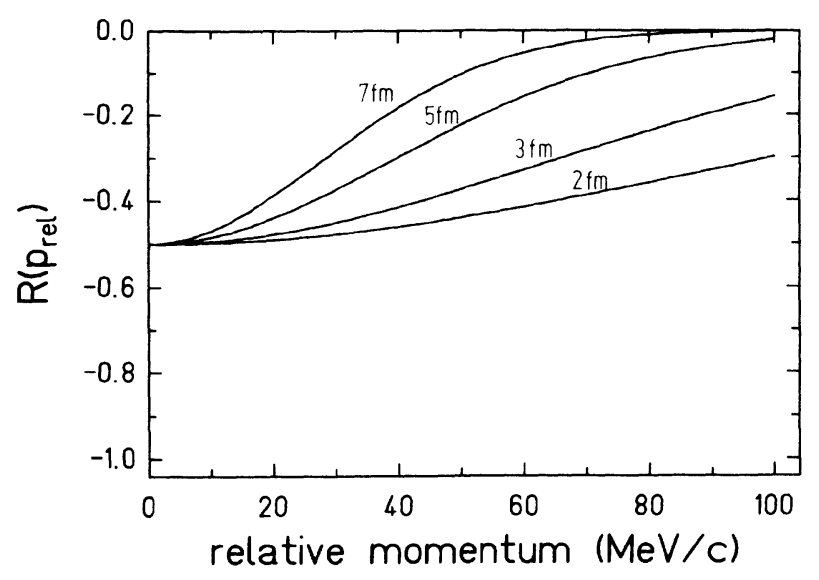

FIG. 14. The two-particle correlation function $R$ of emitted $\Lambda$ 's depends sensitively on the radius $r_{0}$ of the sphere, where the particles are located at freezeout.

$$
R\left(\mathbf{p}_{\mathrm{rel}}\right)=-\frac{1}{2} e^{-\mathbf{p}_{\mathrm{rel}}^{2} r_{0}^{2} / 2} .
$$

Here it is assumed that the two $\Lambda$ 's behave like free particles without low-energy resonance scattering channels. In Fig. 14 one recognizes a sensitive influence of the correlation function $R$ on the radius $r_{0}$. The $\Lambda$ 's are-as discussed above - expected to be emitted from a strongly localized region with radius $r_{0} \sim 2-3 \mathrm{fm}$. The evaporation of particles such as $\pi^{0}$ or protons (and neutrons) should lead to much larger radii $r_{0} \sim 5-7 \mathrm{fm}$. Thus the measured correlation functions of $\Lambda$ 's and pions should correspond to widely differing radii, if strangeness separation does indeed occur. A more detailed study of $\Lambda-\Lambda$ correlations, also including a possible resonance channel, is presently underway. The strangeness separation should not occur for bag constants larger than $235 \mathrm{MeV}$, because associated production would then favor hadronic hyperonic matter and thus actually the hadronic phase would be charged up with strangeness. ${ }^{5,19}$ The dependence of the bag constant, which represents the nonperturbative interactions of QCD, on the density and temperature is unknown.

The creation of (long-lived) droplets of quark matter in high-energy heavy-ion collisions would offer a unique, qualitatively new signal for quark-gluon-plasma formation. It would indeed manifest the existence of a new nonperturbative, high- $\rho$ and $-T$ phase of QCD.

\section{ACKNOWLEDGMENT}

We thank B. Müller for raising the issue of $\Lambda$ correlations.
1J. D. Bjorken and L. D. McLerran, Phys. Rev. D 20, 2353 (1979).

${ }^{2}$ E. Witten, Phys. Rev. D 30, 272 (1984).

${ }^{3}$ E. Farhi and R. L. Jaffe, Phys. Rev. D 30, 2379 (1984).
${ }^{4}$ B. Freedman and L. D. McLerran, Phys. Rev. D 4, 1109 (1978). ${ }^{5}$ C. Greiner, P. Koch, and H. Stöcker, Phys. Rev. Lett. 58, 1825 (1987); Nucl. Phys. A479, 295c (1988); C. Greiner, diploma thesis, Universität Frankfurt am Main, 1988. 
${ }^{6}$ S. A. Chin and A. K. Kerman, Phys. Rev. Lett. 43, 1292 (1979).

${ }^{7}$ D. Vasak, L. Neise, and W. Greiner, Phys. Rev. C 34, 1308 (1986).

${ }^{8}$ R. Hagedorn and J. Rafelski, Phys. Lett. 97B, 180 (1980).

${ }^{9}$ U. Heinz, P. R. Subramanian, H. Stöcker, and W. Greiner, J. Phys. G 12, 1237 (1986); P. R. Subramanian, H. Stöcker, and W. Greiner, Phys. Lett. 137B, 468 (1986).

${ }^{10}$ H. Liu and G. L. Shaw, Phys. Rev. D 30, 1137 (1984).

${ }^{11}$ M. Danos and J. Rafelski, Phys. Rev. D 27, 671 (1983).

${ }^{12}$ B. Banerjee, N. K. Glendenning, and T. Matsui, Phys. Lett. 127B, 453 (1983).

${ }^{13}$ B. Müller and J. M. Eisenberg, Nucl. Phys. A435, 791 (1985); A. Schnabel and J. Rafelski, dipolma thesis (A.S.), Universität
Frankfurt am Main, 1984.

${ }^{14}$ U. Heinz, K. S. Lee, and M. J. Rhoades-Brown, Phys. Rev. Lett. 58, 2292 (1987).

${ }^{15}$ H. Stöcker, G. F. Graebner, J. A. Maruhn, and W. Greiner, Phys. Lett. 95B, 192 (1980); H. Stöcker, Nucl. Phys. A418, 587 (1984); T. Rentzsch et al., Mod. Phys. Lett. A2, 193 (1987); J. D. Bjorken, Phys. Rev. D 27, 140 (1983).

${ }^{16}$ U. Heinz, K. S. Lee, and M. J. Rhoades-Brown, Mod. Phys. Lett. A2, 153 (1987).

${ }^{17}$ D. H. Rischke, B. L. Friman, H. Stöcker, and W. Greiner, J. Phys. G 14, 191 (1988).

${ }^{18}$ S. Koonin, Phys. Lett. 70B, 43 (1977).

19J. Rafelski, Phys. Lett. B 190, 167 (1987). 